\title{
Introduction to the thematic issue on Human-centric computing and intelligent environments
}

\author{
Gordon Hunter ${ }^{\mathrm{a},{ }^{*}}$, Tiina Kymäläinen ${ }^{\mathrm{b}}$ and Raúl Herrera-Acuña ${ }^{\mathrm{c}}$ \\ ${ }^{a}$ Kingston University, London, UK \\ ${ }^{\mathrm{b}}$ VTT Technical Research Centre Ltd., Finland \\ ${ }^{\mathrm{c}}$ Universidad de Tarapacá, Arica, Chile
}

\section{Introduction}

Ambient Intelligence (AmI) and Intelligent environments (IEs) are characterized by information and communication technologies embedded so seamlessly into our physical environments and in various everyday objects that computer-enabled features will become a natural part of our living and working environments [6]. According to Cook and Das [4], the most critical feature that separates intelligent environments from environments that are merely user-controllable is their ability to model inhabitant behaviour. They determine that an intelligent environment is able to acquire and apply knowledge about the environment and its inhabitants in order to improve the inhabitants' experience in that environment. The environment is thus willing to serve spontaneously and proactively, i.e. the environment senses the person's needs and circumstances, and responds accordingly. In general, in the visions promoted by AmI and IEs, people are surrounded by intuitive interfaces and user-adaptive technologies; and the environments are capable of recognizing and proactively responding to the presence of different individuals in a seamless, unobtrusive and invisible way.

Although these visions clearly place humans in the centre of their technology-mediated environments, the human-centred perspective of computer-based systems is still often neglected. Technologists - including computer scientists and engineers - frequently design and construct systems which perform efficiently, but may not provide their users with straightforward modes

*Corresponding author. E-mail: G.Hunter@kingston.ac.uk. for interaction with them, nor a particularly pleasant experience. This can certainly also be the case with AmI and IEs. Coen [2] has critically explicated that in some sense the aim is to make "people interfaces" for computers rather than computer interfaces for people; IE systems use cameras for eyes, microphones for ears and a wide-range of sophisticated sensing technologies to connect with real-world phenomena [2]. Augusto [1] anticipated great challenges in adopting any form of AmI in real use, the chief challenge being that people may not want the technology executing such influential control over their environments. Whilst there do exist such IE systems which are not primarily designed to be used by people, those are the exception rather than the norm. Intelligent buildings, transport systems and cities, robotics, dialogue systems, learning, assisted living and healthcare environments should all have the needs and wishes of their human users as their prime purposes. Cook et al. [3] enumerate the expected benefits as being: increased safety, e.g. by monitoring lifestyle patterns or recent activities and by providing assistance when a potentially harmful situation is developing; comfort, e.g. by adjusting lightning or temperature automatically; and economy, e.g. by controlling the use of electricity and lights [3]. Thus, viewing Intelligent Environments from a human-computer (or human-system) interaction perspective is of vital importance. Consequentially, this special issue of JAISE has HumanCentric Computing and Intelligent Environments as its theme.

Park et al. [9] define Human-Centric Computing as "the discipline that studies the way humans and arti- 
ficial systems interact reciprocally and exchange information", noting that it is closely related to other fields such as sociology, psychology, cognitive science and Human-Computer Interaction (HCI). In [5], Iida and Morita define the aim of Human-Centric Computing as "to build a world in which people and ICT collaborate seamlessly...", with context-aware, multidevice collaboration and human interaction technologies being essential factors. These definitions also resemble the remark from Cook et al. [3] who define ambient intelligence as "a discipline that brings intelligence to our everyday environments and makes those environments sensitive to us". From the usercentric point of view they describe the AmI technologies as being: sensitive, responsive, adaptive, transparent, ubiquitous and intelligent [3]. Park et al. [9] further note that there are two key points of view regarding how Human-Centric Computing and Intelligent Environments are related to each other. From one perspective, IEs should be designed to support their users, so the ways in which those users wish to interact with the IEs, and the ways they receive support from them, must be taken into consideration. From the other viewpoint, IEs usually offer novel technological solutions, such as context-awareness and situationawareness, which then provide opportunities for previously unavailable approaches to the design and implementation of more effective and user-friendly ways for people and the computer-based systems to interact. In essence, the important matters for the users for adapting to the AmI and IE technologies are their usefulness, value, ease of use, sense of being in control, ability to integrate technology into practices - not forgetting trust and social issues of the technologies [6].

Human-Centric Computing has been deliberated in previous JAISE special thematic issues: Volume 4, Issue 5 (September 2012) and Volume 6, Issue 1 (February 2014), and in other previous JAISE articles, e.g. $[7,8,10]$. Recent developments and opportunities have resulted in the interface between Human-Centric Computing and Intelligent Environments being once again highly topical, and hence the initiative for this special thematic issue that emphasizes the human viewpoint: user-friendliness, efficient service support, userempowerment, and support for human interactions in AmI and IEs.

\section{In this thematic issue}

This thematic issue contains a total of six papers relating to different aspects of the theme. In an application-focused paper, "SmartWalker: an intelligent robot walker", Shin et al. describe their development of an intelligent wheeled device to aid the mobility of people, particularly the elderly. Their "walker", equipped with various sensors and actuators connected to a tablet PC, can autonomously navigate around its environment, and provide its user with assistance in an intelligent way. It can also detect people nearby, using a laser range scanner to detect their legs, and locate the user using a face detection system based on Viola and Jones' method [11]. Their system can also recognise gestures made by its user, for example to indicate that the user wants the walker to come over to him/her, or to go back to its base station. Their walker was evaluated using a control group of relatively young adults and a test group of elderly residents of retirement homes.

Three papers relate to the automated recognition of human activities. Chahuara et al., in their paper "On-line human activity recognition from audio and home automation sensors: comparison of sequential and non-sequential models in realistic Smart Homes", compare three sequence-based models (a Hidden Markov Model, Conditional Random Fields, and a sequential Markov Logic Network) with three non-sequence models (a Support Vector Machine, a Random Forest of Decision Trees and a nonsequential Markov Logic Network) for the automatic recognition of human activities of daily living based on evidence from a variety of sensors, including presence sensors, temperature and hygrometry sensors, cameras, door contact sensors and microphones, in a smart home to classify various standard activities of people living in such homes.

Similarly, in their paper "A Novel Approach for Real-Time Learning and Recognition of Human Activities", León, Cuéllar and Delgado describe their study in which the performance of a method based on use of a time series of postures is compared with that of an HMM when applied to the tasks of learning and then recognising a set of human activities associated with everyday living.

Akl et al. employ a method based on a Poisson distribution to model room occupancy, k-means clustering of behavioural types, Kullback-Liebler Divergence to compare activity distributions and "affinity propagation" to compare the behavioural profiles of people who were "cognitively intact" or who had either amnesiac or non-amnesiac mild cognitive impairments in their paper "Clustering Home Activity Distributions for Automatic Detection of Mild Cognitive Impairment in Older Adults". 
Liu et al., in their paper "An Automatic in-home fall detection system using Doppler radar signatures", apply a Doppler radar sensor, and Fourier and Mel Cepstral based features with a Support Vector Machine to identify incidents of elderly people falling at home. They tested and evaluated their system under both laboratory conditions and in a real residence for senior citizens, obtaining excellent detection rates of genuine falls whilst keeping the incidence rate of false alarms at acceptable levels.

Finally, the often-neglected themes of security and privacy of data collected from and used by highly distributed systems such as Intelligent Environments and any system exploiting the Internet of Things (IoT) are addressed by Preuveneers and Joosen in their paper "Security and Privacy Controls for Streaming Data in Extended Intelligent Environments". The authors present their framework SparkXS, integrated with their previously-published SAMURAI lambda architecture, to provide scalable access and data protection controls for the various types, high volumes and rates of data required to be streamed in IoT systems, and in Extended Intelligent Environments in particular. Their framework is designed to meet upcoming EU and other legal requirements, using a "Privacy by Design" approach to ensure that the resulting systems offer users improved control of their own data, and the authors' empirical results on various use case scenarios with large sets of data indicate that their framework is scalable whilst still maintaining acceptable levels of performance.

\section{Acknowledgements}

We would like to thank all the authors for their interesting and high-quality papers, enabling us to put together a coherent thematic issue on this topic. We are also very grateful to the reviewers for their conscientious efforts in evaluating all the papers considered, and giving highly constructive feedback to the authors.
Finally, we would also like to thank the general editors of this issue, Carles Gomez Montenegro and Andrea Prati, for their encouragement and support of our efforts in producing this special thematic issue of JAISE.

\section{References}

[1] J.C. Augusto, Ambient Intelligence: The Confluence of Ubiquitous/Pervasive Computing and Artificial Intelligence, Springer, London, 2007, pp. 213-234.

[2] M. Coen, The future of human-computer interaction, or how I learned to stop worrying and love my intelligent room, IEEE Intelligent Systems \& Their Applications 14(2) (1999), 8-10. doi:10.1109/5254.757622.

[3] D. Cook, J. Augusto and V. Jakkula, Ambient intelligence: Technologies, applications, and opportunities, Pervasive and Mobile Computing 5(4) (2009), 277-298. doi:10.1016/j.pmcj. 2009.04.001.

[4] D. Cook and S. Das, Smart Environments: Technology, Protocols and Applications, Wiley-Interscience, Hoboken, 2004.

[5] I. Iida and T. Morita, Overview of human-centric computing, Fujitsu Science and Technology Journal 48(2) (2012), 124128.

[6] E. Kaasinen, T. Kymäläinen, M. Niemelä, T. Olsson, M. Kanerva and V. Ikonen, A user-centric view of intelligent environments: User expectations, user experience and user role in building intelligent environments, Computers (2012), Special Issue: Large Scale Intelligent Environments.

[7] T. Kymäläinen, The design methodology for studying smart but complex do-it-yourself experiences, JAISE 7(6) (2015), 849860.

[8] T. Olsson, T. Kärkkäinen, E. Lagerstam and L. VentäOlkkonen, User evaluation of mobile augmented reality scenarios, JAISE 4(1) (2012), 29-47.

[9] J.J. Park, A. Coronato, H. Chang and A. Kusiak, Introduction to the thematic issue on ambient and smart component technologies for human centric computing, JAISE 6(1) (2014), 34.

[10] E. Steen, T. Frenken, M. Eichelberg, M. Frenken and A. Hein, Modeling individual healthy behavior using home automation sensor data: Results from a field trial, JAISE 5(5) (2013), 503523.

[11] P. Viola and M. Jones, Rapid object detection using a boosted cascade of simple features, in: IEEE Conference on Computer Vision and Pattern Recognition (CVPR), 2001. 